\title{
From Rural to Urban Environments: Human/Systems Simulation Research for Low Altitude UAS Traffic Management (UTM)
}

\author{
Thomas Prevot ${ }^{1}$, Jeffrey Homola ${ }^{2}$ and Joey Mercer ${ }^{3}$ \\ NASA Ames Research Center, Moffett Field, CA, 94035
}

\begin{abstract}
This paper describes the human/systems simulation research within NASA's UAS Traffic Management (UTM) project. The paper starts with a short description of the UTM project, then presents the UTM development schedule briefly, before leading into more detailed discussions of the simulation test bed's current capabilities, as well as the ongoing and planned human/systems research activities.
\end{abstract}

\section{Nomenclature}

$\begin{array}{ll}\text { ADRS } & =\text { Aeronautical Datalink and Radar Simulator } \\ \text { ANSP } & =\text { Air Navigation Service Provider } \\ \text { ATM } & =\text { Air Traffic Management } \\ \text { ATD } & =\text { ATM Technology Demonstration } \\ E R A M & =\text { En Route Automation Modernization } \\ E T A & =\text { Estimated Time of Arrival } \\ \text { Kts } & =\text { Knots (nautical miles per hour) } \\ L V C & =\text { Live, Virtual, Constructive } \\ M A C S & =\text { Multi Aircraft Control System } \\ N M & =\text { Nautical Miles } \\ N A S & =\text { National Airspace System } \\ R M S & =\text { Root Mean Square } \\ R T T & =\text { Research Transition Team } \\ T C L & =\text { Technical Capability Level } \\ T R A C O N & =\text { Terminal Radar Approach Control } \\ U A S & =\text { Unmanned Aircraft System } \\ U T M & =\text { UAS Traffic Management } \\ U T M-R P & =\text { UTM Research Platform } \\ V L O S & =\text { Visual Line of Sight }\end{array}$

\section{Introduction}

M any beneficial civilian applications of commercial and public Unmanned Aerial Systems (UAS) in lowaltitude airspace have been proposed. Example applications include infrastructure monitoring, precision agriculture, public safety, disaster relief, search and rescue, weather monitoring, news gathering, mapping, entertainment, and delivery of goods. However, none of these UAS operations can currently be conducted in the National Airspace System (NAS) without specific authorizations or exemptions. This is true for uncontrolled airspace (Class G), in which air traffic services are currently not provided, as well as in controlled airspace (Classes A-E). The goal of NASA's UAS Traffic Management (UTM) research is to develop and evaluate concepts and technologies that can safely enable large-scale small UAS operations in low-altitude airspace. ${ }^{1}$

NASA and the FAA are working together as a Research Transition Team (RTT) to determine the architectures and concepts, information flows, and performance characteristics that can enable safe and efficient UAS operations.

\footnotetext{
${ }^{1}$ Research Engineer, Human-Systems Integration Division, NASA ARC Mail Stop 262-4, AIAA Senior Member.

${ }^{2}$ Research Psychologist, Human-Systems Integration Division, NASA ARC Mail Stop 262-4, AIAA Member.

${ }^{3}$ Research Psychologist, Human-Systems Integration Division, NASA ARC Mail Stop 262-4, AIAA Member.
} 
NASA and the FAA are partnering with many additional stakeholders throughout federal, state and local government, industry, and academia, to cooperatively lay out the characteristics of UAS operator roles and capabilities and their support services, as well as the air navigation service provider (ANSP) functions required for UTM. NASA is also spearheading the development of a UTM research platform that instantiates application programming interface (API)-based coordination of UAS operations and services into a research software environment. UTM project release agreements are in place to enable the sharing of certain research software components with the partners. NASA uses the research platform with its partners to test and evaluate increasingly complex UAS operations and associated UTM technical capability levels (TCL). The research results at each TCL provide insight and guidance into concepts and technologies for the respective UTM eco-system and use-cases, and will be a central part of the research transition products generated by the NASA/FAA RTT.

The test and evaluation of increasingly complex operations requires the capability to generate rural, suburban and urban operations at various fidelity levels, including live operations, virtual human-in-the-loop simulations, and constructive autonomous batch modes. This live, virtual, constructive (LVC) environment enables the safe progression from low-density, low-risk (e.g. rural), to medium-risk (e.g. suburban) and high-risk (e.g. urban) environments. While low-risk, small-scale operations can be tested to some degree at UAS field sites, complex operations, such as urban operations and large-scale contingencies, will be first simulated in the lab environment and supplemented by a few field operations. This paper will provide an overview of the UTM simulation research, including the simulation and LVC capabilities, and then describe the human/systems aspects that are addressed in this environment.

\section{UTM Research Technical Capability Levels}

Each capability is targeted to specific types of applications, geographical areas, and use cases that represent certain risk levels. The pace of development targets a new UTM TCL every 12-18 months to be tested and evaluated in simulation and field trials. Figure 1 summarizes these capabilities and the test schedule.
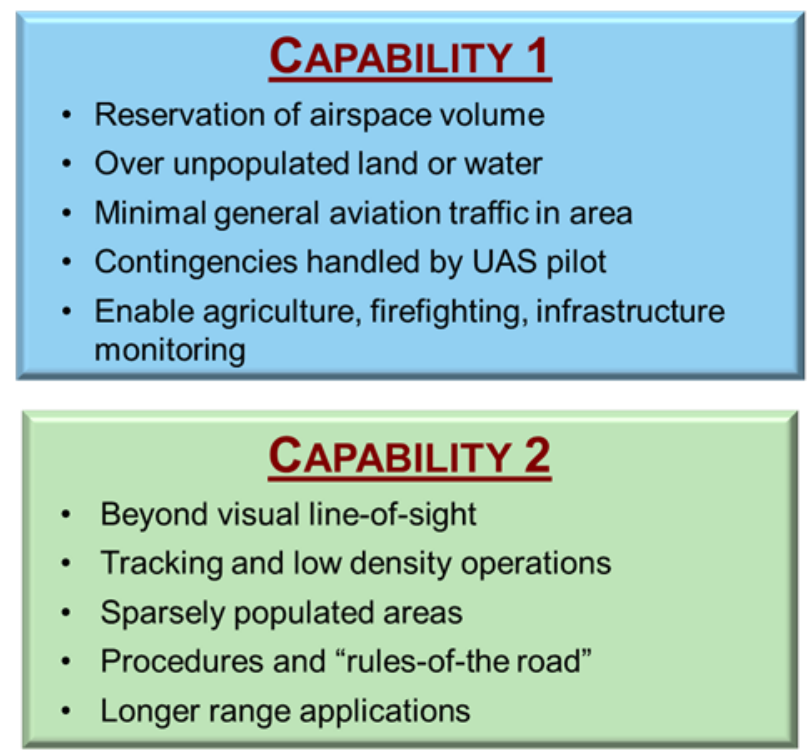

Figure 1 UTM Research Technical Capability Levels

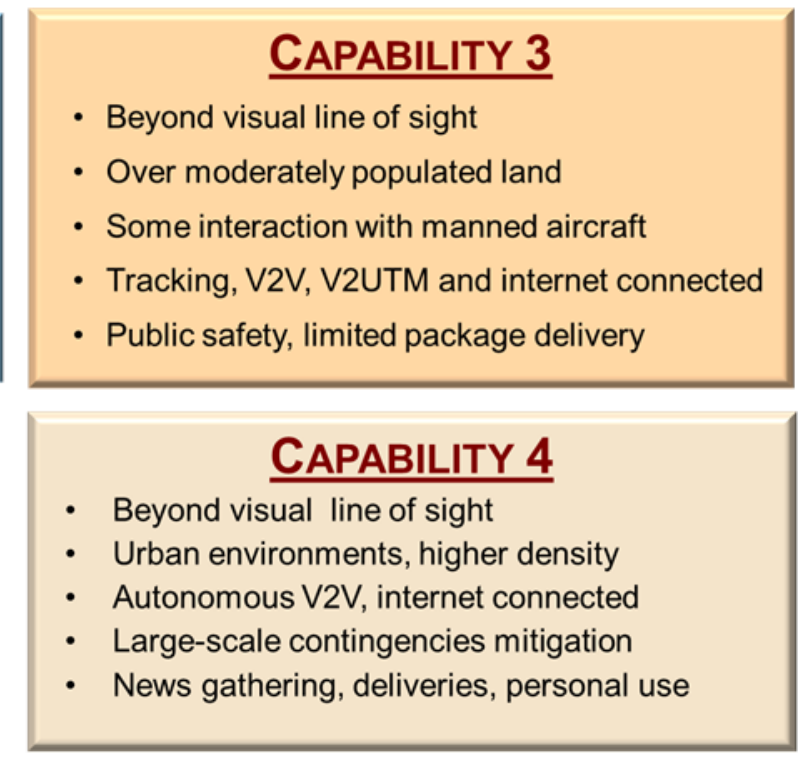

The tests are joint efforts involving NASA and its government, industry and academic partners. NASA assumes primary responsibility for the test coordination, conduct and data analysis and the development of the supporting UTM research platform and its associated APIs and Interface Control Documents (ICDs). Its partners provide vehicles, mission scenarios, advanced data services, surveillance assets, and additional supporting technologies that interoperate with the core UTM research platform.

Capability 1 has been field tested in August 20162015 at Crows Landing Airport in California and also at the six FAA UAS test sites in April 2016 where all sites conducted UAS operations concurrently.. It provided interactive planning and constraint management capabilities to manage multiple UAS operations in low risk rural areas within visual line of sight (VLOS). The field demonstration details and results will be published in a separate report. 
Capability 1 used a simple airspace reservation system to segregate UAS operations with geo-fences in areas of low risk to people and property on the ground and provides user authentication and vehicle registration services. Displays and mobile applications created for the capability were made available to the FAA UAS test sites for further use and evaluation. Capability 2 will extend capability 1 to support beyond visual line of sight (BVLOS) operations and permits increased traffic density by allowing segmented and altitude separated flight plans. The focus will be the development of procedural rules-of-the-road to maintain the safety of beyond line-of-sight operations when UAS operations share airspace. Contingency management will be automated for individual vehicles. UTM research capability 3 will extend capability 2 to permit UAS operations in the vicinity of manned aircraft over moderately populated areas. The focus will be the development of in-flight separation services, trajectory conformance monitoring, and automated contingency management involving multiple vehicles. Capability 4 will include the ability to handle large-scale contingencies involving all UAS vehicles simultaneously. The focus will be the development of procedures and technologies to handle "all land" scenarios or widespread surveillance outages

\section{UTM Test Environment}

UTM research and development has a strong focus on field demonstrations of actual operations and capabilities. The goal is to accelerate airspace access for the new entrants and their use-cases, including public safety, first responders, deliveries of medicines and consumer products, photography and many more. However, field activities are tightly regulated and even after extensive review processes do not allow complex BVLOS operations in many of the environments that are of primary interest for most operators, such as suburban and urban environments. Therefore, NASA is creating a test environment that enables simulation of all these use-cases in order to develop the requirements, procedures, system capabilities and interfaces that may enable complex operations. The initial UTM test environment is implemented as a UTM laboratory in the Airspace Operations Laboratory (AOL) ${ }^{1}$ at NASA Ames Research Center.

\section{A. UTM laboratory}

The current UTM laboratory is comprised of multiple test areas and a distributed network of client stations, servers, and display media. The test areas where development work is concentrated consist of multiple workstations that are equipped to fulfill various UTM-related roles, and are configurable to suit a variety of situations. Although the entirety of the Airspace Operations Laboratory can be leveraged for UTM related research, there are generally two main test areas that are used for simulation and demonstration. The first of these test areas consists of a cluster of workstations that are predominantly used for scenario development, software testing, and procedure preparation. This area can host a number of researchers working in close proximity, and provides the ability to simulate multiple UTM operations from multiple clients. Researchers are also able to gain an awareness and understanding of those operations in the context of the larger operational environment through desktop and mobile applications on workstations and mobile devices in this test area. Additionally, to increase the fidelity of simulated operations and assist in the development of test procedures, laptops equipped with the same Mission Planner [reference] and NASA-built UTM client software used during field trials are also available in this test area.

Given its location and layout, the other test area is often used for providing concept and capability demonstrations. Similar to the test area previously described, this area consists of a number of workstations that are able to connect to the UTM server and simulate operations. Desktop and mobile applications are also available for providing an operational overview as seen in the Figure 2. Opposite the workstations in this test area is a large

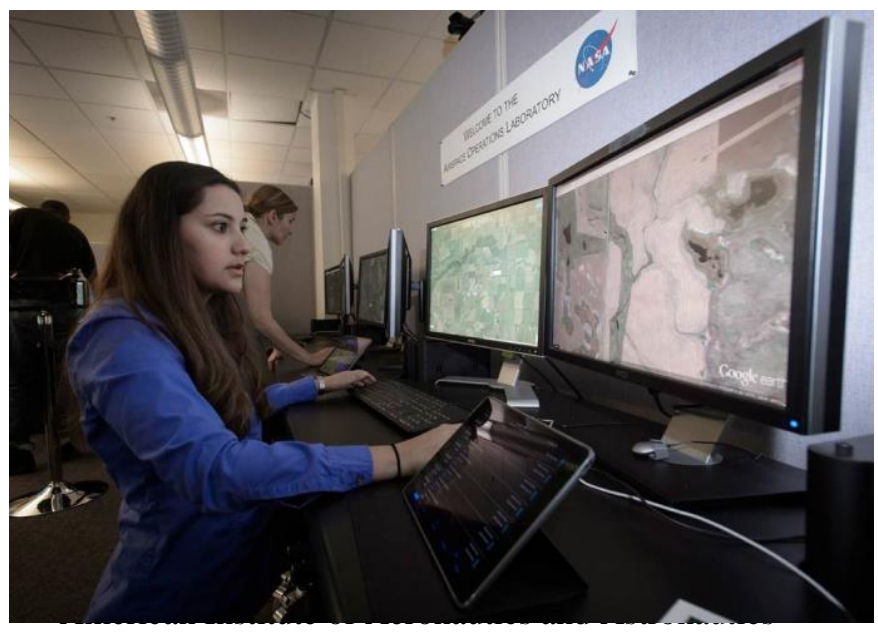

Figure 2. Demonstration test area 
display wall that is capable of showing 12 independent content sources, driven by workstation or app displays, and can be configured for many different arrangments and combinations. Figure 3 presents the layout of the display wall as it was used in support of the National Campaign event. In the figure, the left half of the display wall shows the map displays of the desktop application for each of the six test sites that were involved in the event. During operations, each of the test site's map display showed all of the active operations at their site, giving researchers an integrated overview of the distributed operations. The right half of the display wall shows a mirrored view of what is being shown on a mobile application running on two separate tablets. The four rightmost display panels show Google Earth displays from the viewpoint of specific aircraft in the environment in which they are flying. Any vehicle that is reporting positions within the UTM environment can be displayed in such a manner via a "push-towall" function in the mobile application and links to a Gateway application developed for the purposes of viewing operations. These applications will be described in greater detail in section IV. Figure 4 presents a closer view of the mirrored tablet application and the "push-to-wall" capability. It should be noted that although the test areas have been described separately, the components that make up each area (with the exception of the display wall) and the underlying system are the same.

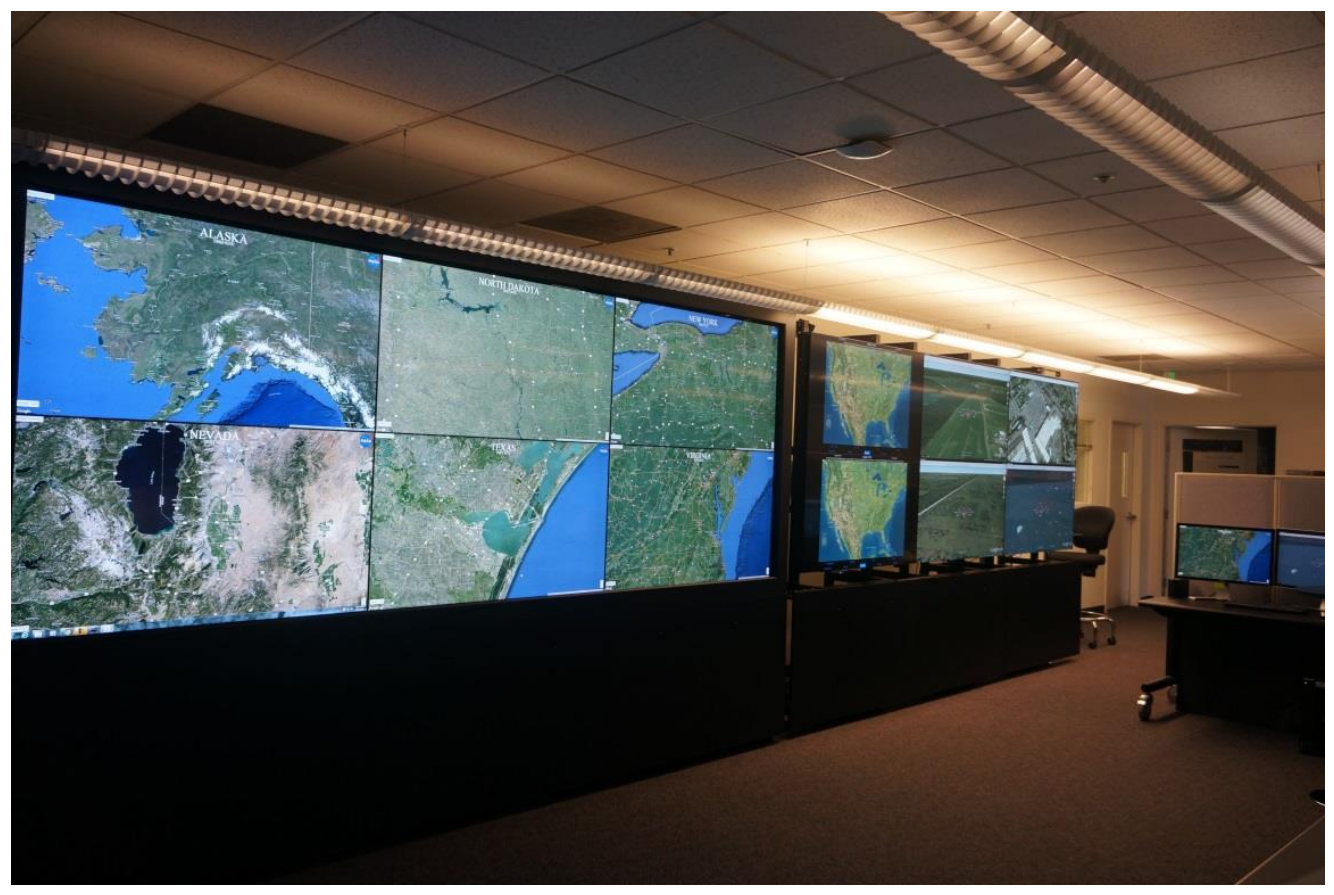

Figure 3. Display wall in demonstration area 


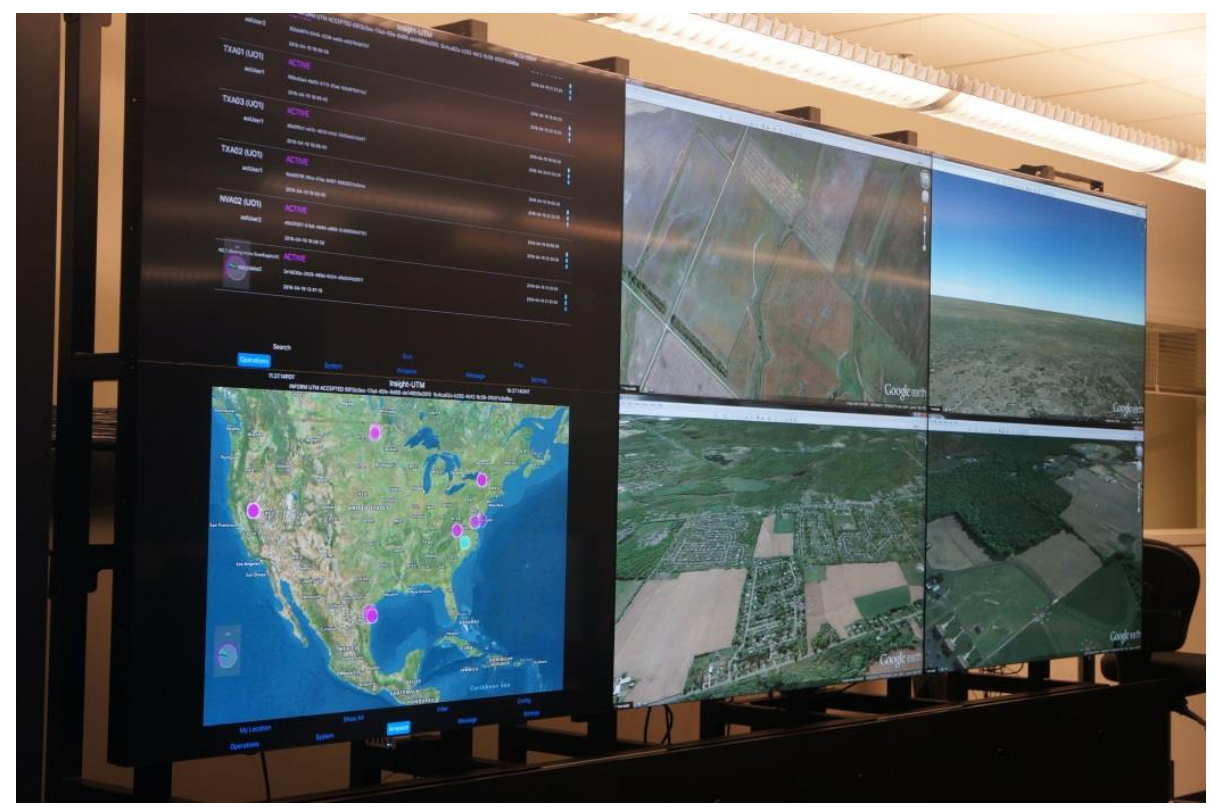

Figure 4. Closer view of mobile application and "push-to-wall" displays

\section{B. Components and Information Flow}

The simulation infrastructure has been designed to reflect the components and architecture of the current, outward-facing system, and also serves as an internal testing infrastructure that provides the ability to scale to future environments. Figure 5 presents the basic architecture of the simulation environment with the associated components and flow of information. At the core of the simulation infrastructure lies the UTM server, which hosts the software and services developed for UTM operations. At a high level, the software and services can: receive operation plan proposals from operator clients; perform constraint checking of those plans against static constraints (e.g., airports, airspace classes, national parks), other active and proposed operations, and temporary restrictions; and receive position updates sent by active vehicles, and check those position locations to ensure that the operation is conforming to its approved boundaries. The server provides state change messages that indicate an operation's transition from one state (e.g., 'Active') to another (e.g., 'Closed'), as well as messaging from operator clients into the system in the form of free text or UTM Reports (UREPs), all of which are consumable by other connected clients with the proper credentials.. Automatic broadcast messaging from the system to appropriate clients is also included for off-nominal situations.

The UTM laboratory is able to connect its simulation components to a number of different UTM servers with minimal changes required. This ability allows for the demonstration of existing capabilities that have been included in, for example, the flight demonstrations for Capability 1 (2015), while being able to connect to separate servers that are in development for Capability 2 and beyond. 


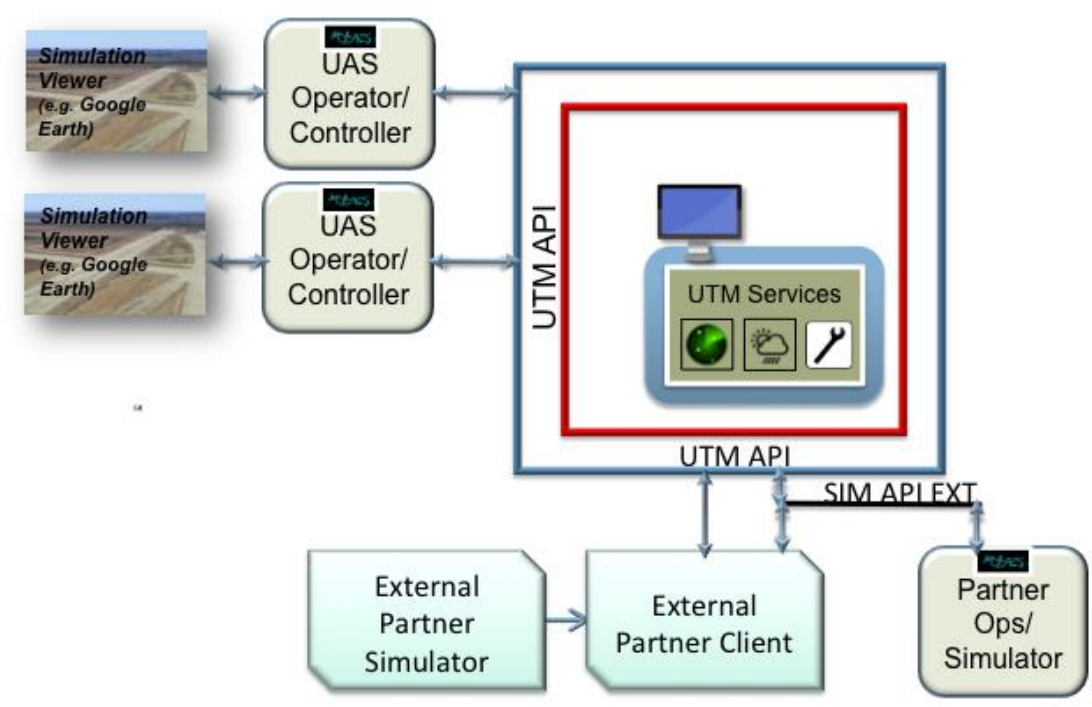

Figure 5. Schematic of simulation infrastructure

Operators connect to the UTM server via the published UTM Application Programming Interface (API). In general, there are two types of clients that are actively developed in the laboratory for different operator types. The first type of client is associated with simulated vehicle operations. For the simulation of UAS vehicle operations within the UTM environment, NASA's Multi-Aircraft Control System (MACS) ${ }^{2}$ has been further developed to serve as a UAS operator interface and to include flight dynamics that more closely emulate the behavior of small multirotor and fixed wing aircraft. Part of the operator interface includes the capability to develop operational plans and flight profiles that can then be submitted to the UTM server and flown autonomously according to its plan. Vehicle controls are also available for the operator to manually fly the aircraft when necessary. Figure 6 presents a screen capture of the MACS operator station and client interface, which includes the vehicle control panel, map overview display, route display, and UTM message window. The message window shown is the front end of the operator client interface that displays the messages to and from the UTM server regarding each flight under the operator station's control. For explanation, the messages shown in Figure 6 indicate that the operator has a valid connection to the UTM server, the operational plan for a flight has been sent, the plan has been checked and subsequently accepted, and the flight has transitioned to an activated state. The number of operator stations within the UTM laboratory available to connect to the UTM server are only limited to the number of workstations available. Additionally, each MACS operator station is capable of controlling one or multiple vehicles. 


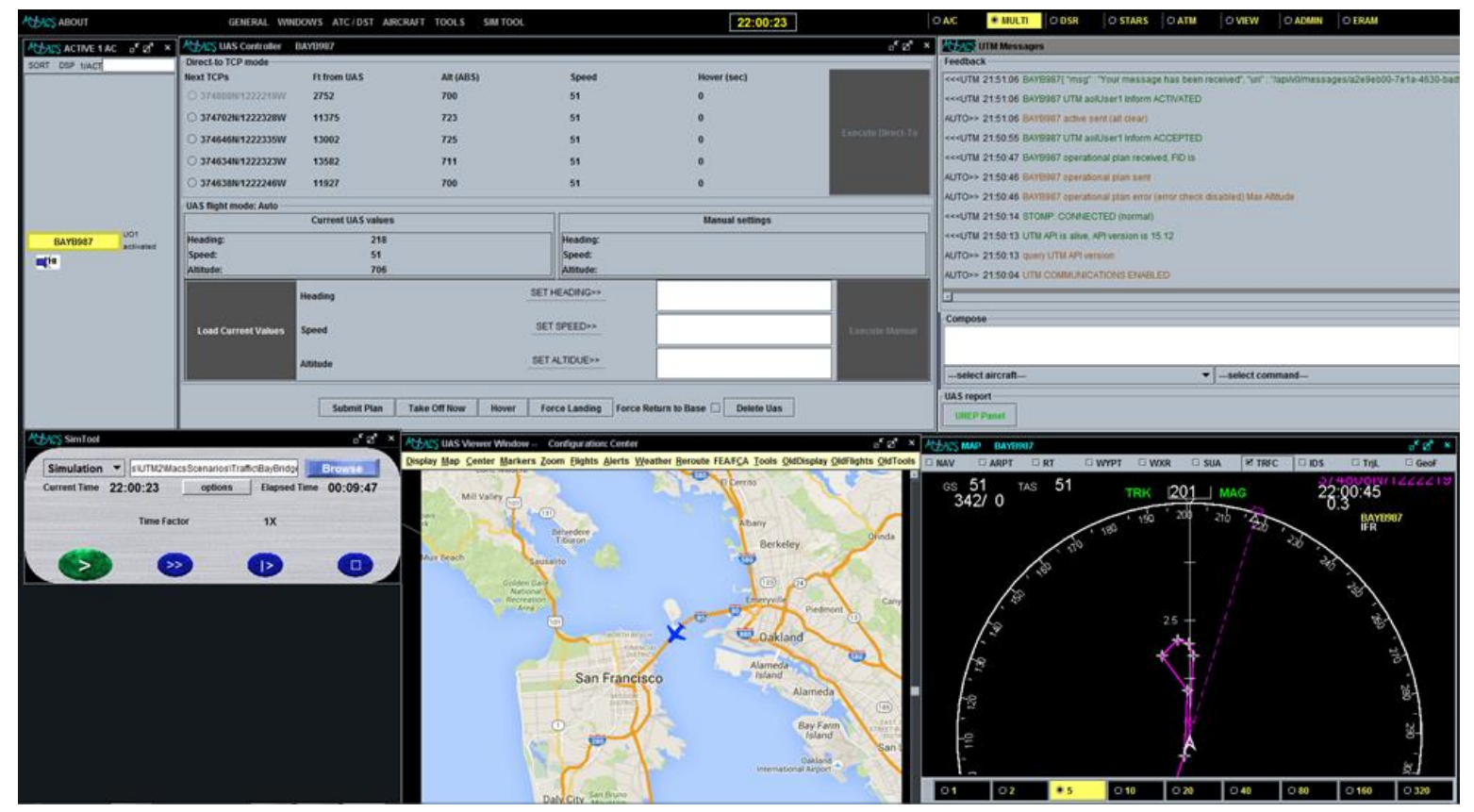

Figure 6. MACS operator station

An additional feature of the MACS operator station that has been developed is a connection to the Google Earth application. This connection provides inputs from the MACS station and allows for the real-time 3D visualization of vehicle location and movement in the environment in which it is flying. The viewing angles and distances are all configurable and able to be changed at any time. Given that there is a direct connection between the MACS operator station and the Google Earth interface, all of the vehicles under the control of the operator station are available for viewing. The operator can cycle through all of the active flights and the display will update to reflect the selection. The vehicle models rendered within Google Earth are also able to be tied to the associated vehicle type flown in MACS such that, for example, if the vehicle being flown in MACS is a quad-rotor vehicle, when selected for viewing it will be displayed as a quad-rotor vehicle. Figure 7 presents an example display where an octo-rotor vehicle being flown in MACS (see Figure 6) is rendered in Google Earth as it proceeds on an infrastructure inspection of the Bay Bridge leading into San Francisco, CA.

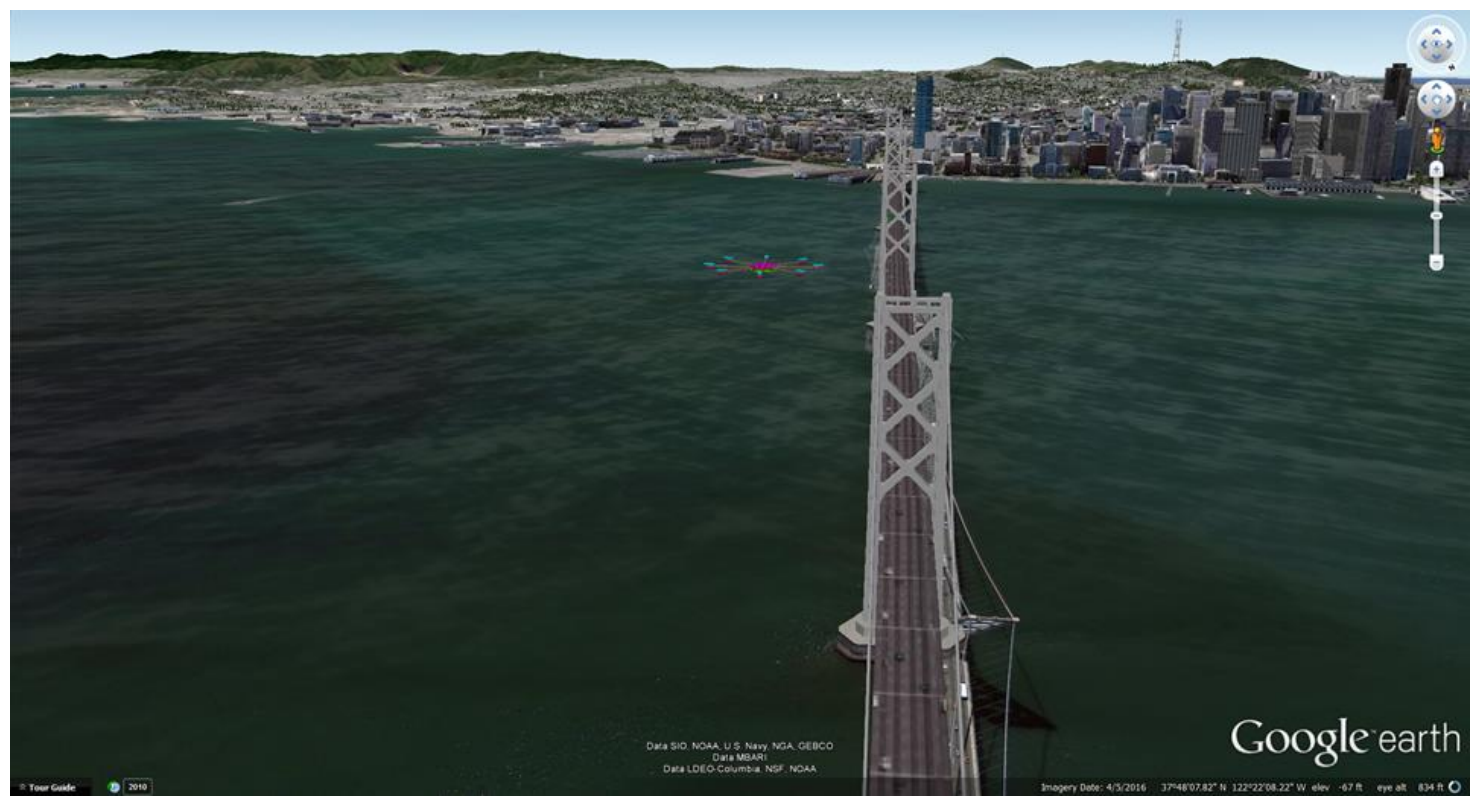

Figure 7. Google Earth visualization of vehicle flown in MACS

American Institute of Aeronautics and Astronautics 
Another operator client that is available for use in the UTM laboratory is one that was developed specifically for actual flight tests in the field. While the MACS operator client is tightly integrated with the software, this additional client is more stand-alone and tailored for single vehicle operations. The client is able to read in waypoint files designed using the Mission Planner interface and submit operational plans to the UTM system based on the flight profile. A connection between this client and the Mission Planner software allows for the simulation of flights starting from plan submission, throughout the flight phase, and to operation closure. Manual control of the simulated flights is also facilitated by the control capabilities included in Mission Planner. Although this client was not originally developed for laboratory use, the open architecture of the simulation environment allows for the integration of almost any client developed in accordance with the published ICDs.

Clients for UTM Management applications are also in development within the UTM laboratory. These applications are designed to provide situation awareness of operations within the system and also facilitate the interaction of appropriately credentialed individuals to interact with the system and flights for specific purposes (e.g., reserving an area of flight for first responders). There are two types of interfaces in development to suit these needs: a desktop application that can be run from any workstation within the laboratory, and a mobile iOS application that can be used on any appropriately provisioned Apple iPad or iPhone. These interfaces will be described in greater depth in section IV.

\section{UTM laboratory activities}

Given the infrastructure and capabilities of the UTM laboratory, the use cases that it can be applied to are numerous. There are currently four broad categories where the laboratory is used extensively. The first of those is the development and testing of use cases in current and future environments. The scenario development capabilities in MACS allows for the exploration of almost any type of use case that is currently being envisioned for practical application. The integrated architecture also allows researchers to gain insight into how the many varied types of operations will be able to be accommodated in the same UTM environment and step those through the different capability levels referred to in Figure 1. The ability to apply the different use cases to different future capability levels is an important aspect of the UTM laboratory's capabilities in that researchers and planners can get a glimpse into potential UTM operations that are not possible to test in today's environment due to safety considerations (e.g., dense operations in urban environments).

Another area in which the UTM laboratory is heavily involved in is software testing. Although the development teams have internal tests that ensure basic functional requirements are met, extensive testing within the laboratory is also performed throughout the development process. Due to the different types of operator and management applications available within the laboratory, and the ability to tailor test cases to target specific functionalities, software testing in the laboratory has proved critical in determining the readiness of software to be deployed for live flight demonstrations. Similarly, prior to any flight demonstration, the laboratory is used to step through each of the test cases scheduled for actual flight testing in order to ensure that the procedures have been designed appropriately, and that the software on the server and client sides are all functioning as intended.

In addition to serving as a testing environment prior to flight tests, the UTM laboratory has been directly involved in many of the live flight tests conducted thus far as a Live, Virtual, Constructive (LVC) component. The logistical and safety constraints of live operations in the field tests often limit some of the types of testing that can be performed. Examples of such cases include more dense operations to test messaging capabilities, and off nominal events that would be unsafe to test with live vehicles. The infrastructure and development capabilities of the laboratory's components enable the instantiation of simulated operations into the UTM environment to operate simultaneously alongside live vehicle operations. Within the system, there is no functional difference between simulated and live operations: each flies according to their submitted operational plans and provides position updates throughout, and each does so by communicating to the UTM system using the same message formats. The ability to insert simulated flights into a live test environment increases the number and types of cases available for investigation.

The final area to be described that the UTM laboratory is involved in is what is referred to as load testing. This type of testing examines the capacity of the UTM server, related infrastructure, and the different operator and management applications to handle incrementally increasing numbers and types of operations and operator connections. The process of load testing serves to identify the limits at which the system as a whole breaks down, which provides insight into how different configurations and approaches can mitigate such events and provide a path to a more robust and adaptive system. 


\section{Human/Systems Research}

One of the primary purposes of the simulation test bed is to facilitate rapid prototyping and evaluating of the human/systems interfaces and procedures that are required for the early field demonstrations and the build deliveries to partners. At the present time a desktop application and an iOS application have been developed and used for the technical capability 1 testing. By offering map, list, and timeline views, these applications support the needs of a wide range of UTM users.

\section{A. Desktop UTM application}

The AOL's desktop UTM application was originally developed for the ability to monitor and supervise the activities of the early field tests. The resulting design was a simple, two-window layout that included a time-based list view and a map view (see Figure $\mathrm{x}$ ). The two windows can be arranged and sized to however best suits the user. Many of the workstations in the AOL are connected to two monitors, and a common arrangement for the desktop application is to maximize each window devoting one monitor to each. Such an arrangement is shown in Figure x, with the left side depicting the list view. The list view provides access in a tabular format to all of the current operations known to the UTM system. The user can then sort and filter those operations by several parameters, such as time, ID, or the operation's current state. The list view is supplemented by a timeline and messaging consoles. The timeline's 'current time indicator line' and 'mission block' graphics help to convey the temporal length of operations, as well as their timing relative to the current time and to each other. Messaging consoles in the list view give the operator the ability to see system messages and to communicate with one or multiple aircraft operators. For users with elevated privileges, the system messages regarding constraint violations can be used to submit constraint override commands.

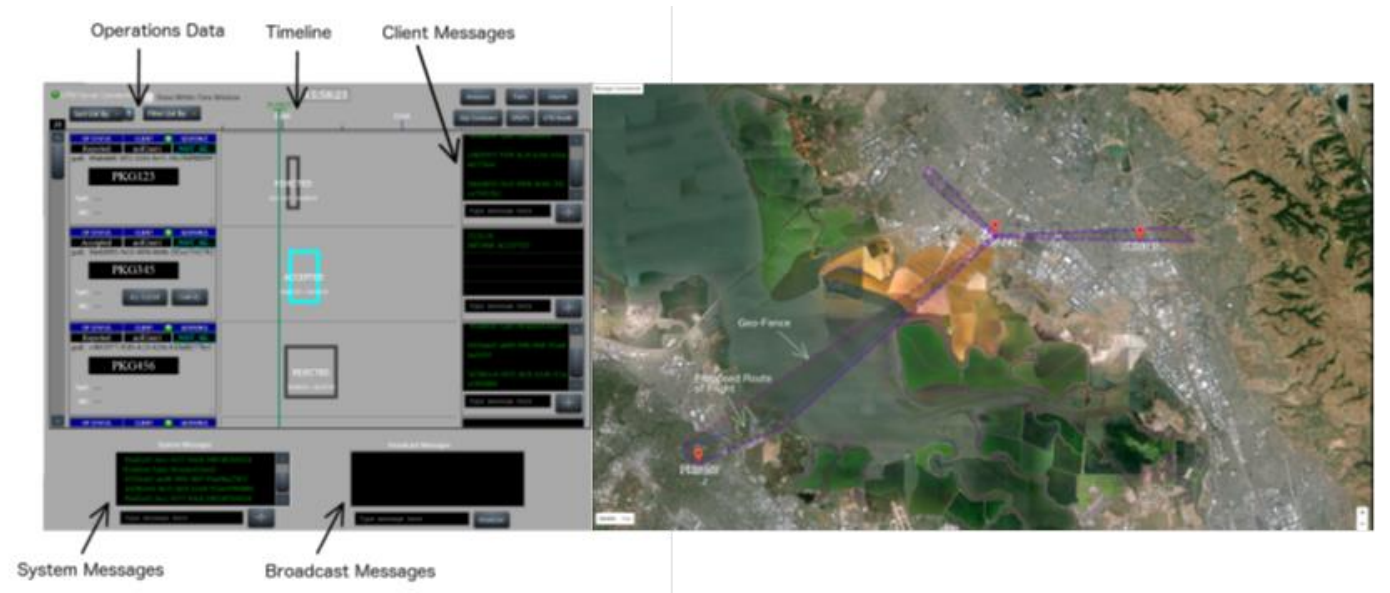

\section{Figure 8. Sample screen layout of desktop UTM application}

The desktop application's map view, shown in the right side of Figure $\mathrm{x}$, is instantiated inside a web browser and overlays the locations of the current operations known to the UTM system onto imagery from Google Maps. Controls to toggle the display of static constraint boundaries are available in the list-view window, allowing the operator to visualize the location of the current operations relative to those constraints (see Figure $\mathrm{x}$ ). In addition to the real-time position of aircraft, the map view can also display an aircraft's submitted route of flight, and the system-allocated geo-fence surrounding that route of flight. The map view also includes tools for drawing polygonshaped constraint areas, that users with elevated privileges could submit to the UTM system (see Figure x). 

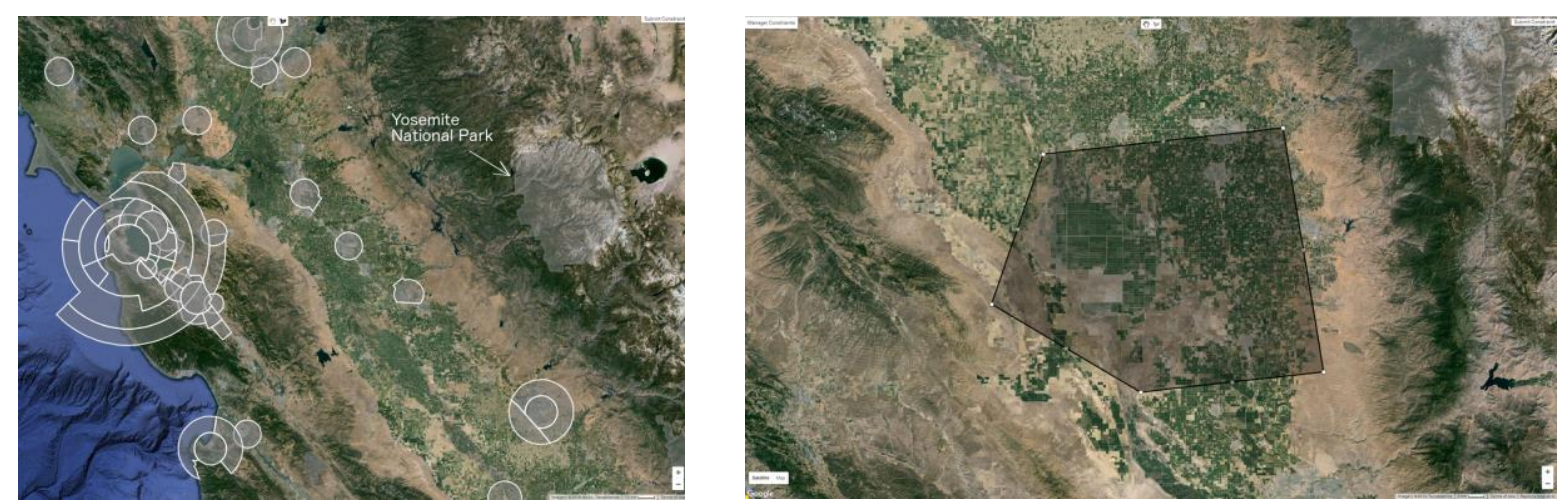

Figure 9. The desktop UTM application's map view showing constraint boundaries (left), and custom constraint editing capabilities (right).

In addition to viewing nominal operations with these displays, the desktop application can support operational scenarios that require additional input. For example, a vehicle operator representing a film and photography company wishing to fly in a national park (after obtaining permission to do so) may request that a system authority override that constraint so that their submitted operation won't get rejected. Similarly, a city's government may want to block all operations along a popular street during a school parade, and might use the desktop application to create a new constraint for the appropriate area and time.

\section{B. Mobile UTM application}

The AOL has also developed a UTM application for the iOS platform. Running this software on portable devices allows the mobile UTM application to support a wide range of use cases; from 'read-only' viewing and gathering of situation awareness, to manager-type functions like those available in the desktop UTM application. The mobile application organizes information into different pages, such as Operations, System, Airspace, Message, and Settings. The System, Message, and Settings pages provide information regarding system health, messaging traffic, and connection settings, respectively. The Operations and Airspace pages are seen in Figure x, and offer a list view and a map view.
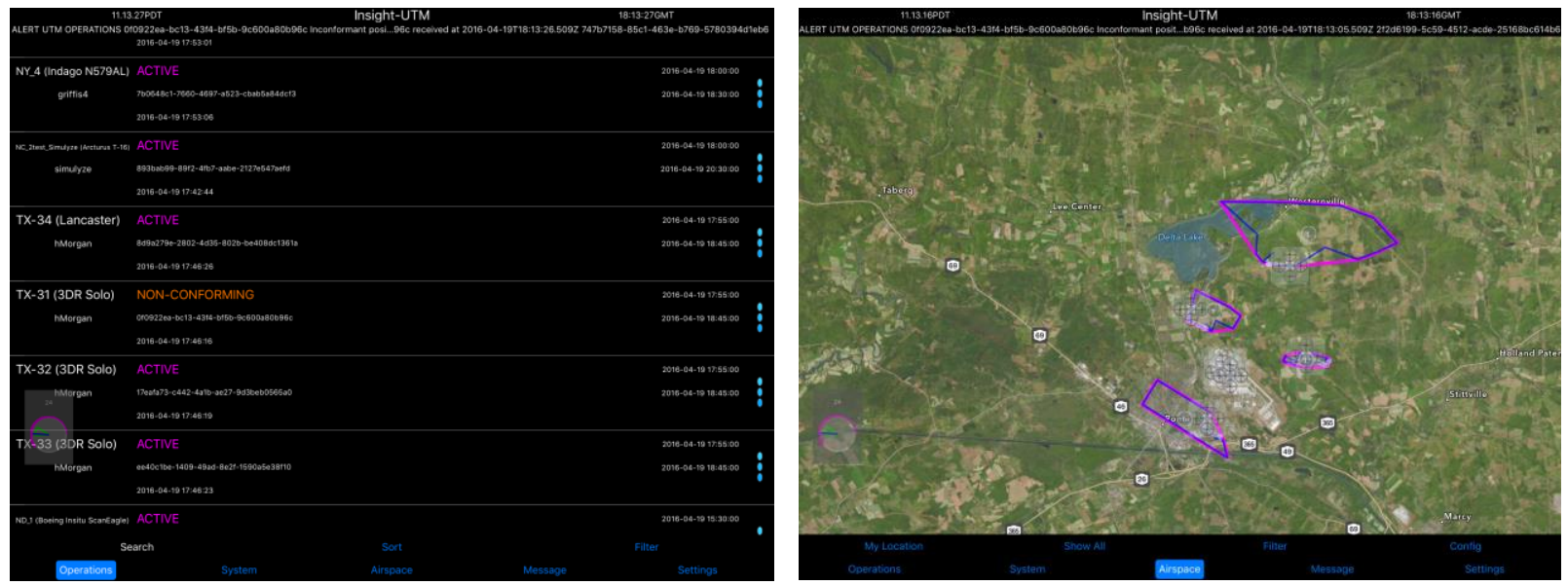

Figure 10. The mobile UTM application's Operations (left) and Airspace pages (right).

Similar to the desktop application's list view, the Operations page is a tabular list of all the current operations known to the UTM system that can then be sorted and filtered. Display elements within the Operations page indicate an operation's ID, state, submission time, as well as its start and end time. The three light-blue dots at the right of an operation's list entry bring up a menu of pre-defined interactions, such as sending messages, and hiding/unhiding operations. Selecting a list entry brings up the 'detailed information' page for that operation, seen in Figure x, which carries over many of the display elements from the Operations page, while adding a zoomed-in 
map view of the operation, its aircraft type, and it's messages log. For users with elevated privileges, the interaction menus include options such as activating an operation (important for a vehicle operator whose plan submission was accepted, and is now ready to take-off), closing an operation (important for a vehicle operator whose operation ended), and unique to the AOL's UTM laboratory, 'pushing' an operation to the video wall. This last feature, when properly configured, allows system analysts to collaborate by sharing what they are viewing on their tablets. For example, and was done during the National Campaign, if an analyst is using the mobile application to follow the activity of a particular operation, and wants to view its real-time position within its surroundings, they can use the 'push-to-wall' feature. By displaying an instance of Google Earth on one of the video wall's monitors, the 'push-towall' feature connects to that Google Earth instance, driving it with the stream of real-time position updates from the aircraft, and displays on the video wall a continuous visualization of the aircraft's flight progress (as seen in Figure 7 , for example).

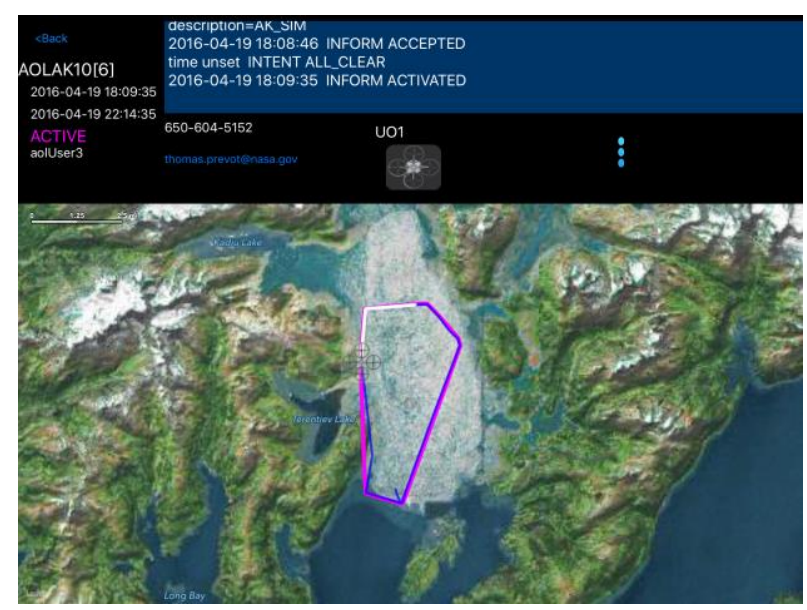

Figure 11. The mobile UTM application's detailed information page for a selected operation.

Similar to the desktop application's map view, the mobile application's Airspace page displays, according to the filter options selected, all of the current operations overlaid onto a mapping platform. For a given operation, the Airspace page includes the vehicle's current location, submitted path of flight, the surrounding system-allocated geo-fence, and 'breadcrumb'-type history trails. The operation's current state (e.g., Active, Closed, etc.) is conveyed in the Airspace view using the same color as the text from the Operations view. Both the Airspace and Operations pages have a clock icon at the lower-left edge of the screen, which the user can tap to bring up time sliders for defining the parameters of the time filter. In addition to the same filter options found in the Operations page, the Airspace page has some quick-access features to help navigate around the map. Buttons are available that center and zoom the map to fit to the screen: the Continental US ('CONUS'), the device's current location ('My Location'), and the area bounding all current operations ('Show All'). A 'Layers' button provides controls for enabling/disabling the display of national parks, class airspaces, airports, and manager constraints, with settings to control their opacity/transparency. To highlight other features of the mobile application, Figure $\mathrm{x}$ shows the Airspace page in two specific scenarios. When showing an area with multiple, overlapping operations, (i.e., fairly zoomed-out), the Airspace page uses a clustering display technique to group operations so as to provide a simplified overview of the locations of many operations. Conversely, when zoomed-in and showing multiple operations, (i.e., dense environments), the Airspace page offers additional display elements that the user can select to see more information about an operation. Selecting an individual aircraft icon will display its ID (callsign and GUFI), and offers buttons to jump directly to that operation's details page, or to push that operation to the video wall. 

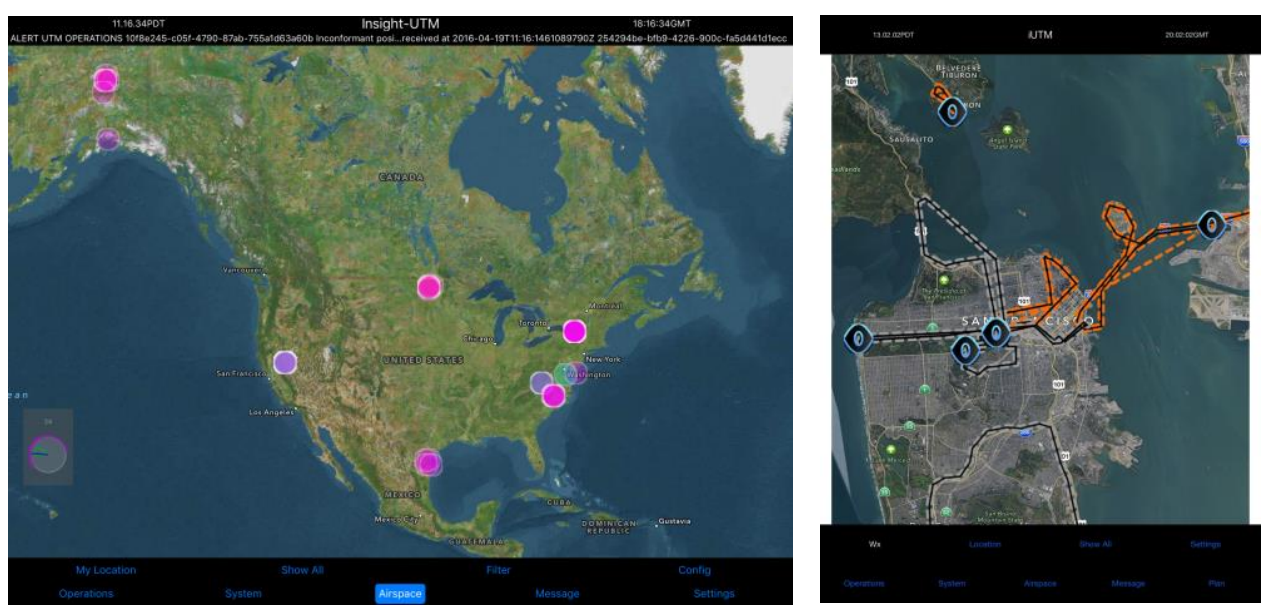

Figure 12. The mobile UTM application's Airspace page displaying operation clusters (left), and a close-up view of urban operations (right).

\section{Interface updates for advance UTM Technical Capability Levels}

In support of BVLOS operations, Capability-2 adds new functionalities that require special consideration for integration with the desktop and mobile applications. Under Capability-2, submitted operations can consist of more than one segment. As an aircraft progresses along its path of flight, this notion of multiple segments allows the UTM system to consider 'past' segments as unoccupied airspace, thereby increasing the capacity for other operations. Considering that Capability-2 operations can also be separated from one another vertically, each segment of an operation now includes specified start- and end-times, and floor and ceiling altitudes. Displaying these traits on a two-dimensional display so that a user can easily understand the traffic picture is important so that users can, for example, identify which vehicles are above or below another in flight-following tasks, or know which constraints a submitted operation violated so as to make the appropriate adjustments.

The later capability levels of UTM also expect denser operations in increasingly urban environments. Early load-tests of the system revealed certain design short-comings when simulating several hundred aircraft. Most obvious was the value of screen real-estate, and the need to convey as much information as possible in as little space as possible. The desktop and mobile application's list views can respectively only fit, respectively, three or seven operations on the screen at one time, clearly demonstrating the need for alternative interface designs.

\section{Future plans}

UTM will include increasingly autonomous operations and systems. As the development progresses the human/systems simulation research will increasingly focus on multi-agent human/autonomy teaming and contingency management and the simulation environment will become much more capable. It is envisioned that NASA and its partners will be testing large scale UAS operations in all environments from rural to urban settings using LVC technologies to continue the development and evaluation of the UTM concepts and technologies

\section{Conclusion}

NASA has developed a powerful human/systems simulation research platform to test the concepts and technologies within NASA's UAS Traffic Management (UTM) project. The sUAS simulation capabilities extend the simulation capabilities that already existed in MACS towards being able to test many diverse small UAS operations and use cases. The UTM laboratory has proven invaluable for verifying and validating the software of the core UTM research platform, pre-testing flight test scenarios, observing actual tests and demonstrating the UTM concepts and research platforms to stakeholders and media. 


\section{Acknowledgments}

We greatly appreciate the hard work of the entire AOL staff and all the folks in the human/systems integration division at NASA Ames Research Center that support our work. Moreover, much of the capabilities and research described here is a result of our close collaboration with many colleagues in the Aviation Systems Division at NASA Ames. We greatly appreciate everybody's support. This research was funded by the Safe Autonomous Systems Operations (SASO) Project of NASA's Airspace Safety and Operations Program.

\section{References}

${ }^{1}$ Kopardekar, P., Rios, J., Prevot, T., Johnson, M., Jung, J. and J.E. Robinson III (2016) Unmanned Aircraft System Traffic Management (UTM) Concept of Operations, AIAA Aviation 2016, Washington DC, June

${ }^{2}$ Prevot, T., Smith, N.M., Palmer, E.A., Callantine, T.J., Lee, P.U., Mercer, J.S., Martin, L.H., Brasil, C. and Cabrall C. D. "An Overview of Current Capabilities and Research Activities in the Airspace Operations Laboratory at NASA Ames Research Center" AIAA 2014-2860, 14th AIAA Aviation Technology, Integration, and Operations Conference, Atlanta GA, June 2014

2 Prevot, T. (2002) Exploring the Many Perspectives of Distributed Air Traffic Management: The Multi-Aircraft Control System MACS. In S. Chatty, J. Hansman, \& G. Boy. (Eds). HCI-Aero 2002, AIAA Press, Menlo Park, CA. pp 149-154, 2002. 